\title{
Applications of the Stochastic Ising Model to the Gibbs States $\star$
}

\author{
Richard A. Holley and Daniel W. Stroock \\ Department of Mathematics, University of Colorado, Boulder, Colorado, USA
}

\begin{abstract}
The stochastic Ising model is used as a tool to prove theorems concerning analyticity of the correlation functions and strong cluster properties of the Gibbs states.
\end{abstract}

\section{Introduction}

The stochastic Ising model has been used as a model for the time evolution of the configuration of spins in the classical Ising model. From a physical point of view the model has the unfortunate feature that the dynamics do not come from a Hamiltonian and are not well motivated. Nevertheless it is possible to learn something about a Gibbs state by studying the semi-group of the stochastic Ising model which has that Gibbs state as its stationary measure. The results proved in this paper demonstrate this technique.

Let $Z^{d}$ be the $d$-dimensional integer lattice and let $\left\{J_{R}: R\right.$ a finite subset of $\left.Z^{d}\right\}$ be a potential which satisfies

(0.1) $J_{R}=J_{R+k}$ for all $R \subset Z^{d}$ and $k \in Z^{d}$

and

(0.2) $\sum_{R \ni 0}\left|J_{R}\right|<\infty$.

Let $E=\{-1,1\}^{Z^{d}}$ be the set of configurations of spins and give $E$ the product topology. The elements of $E$ are denoted by letters such as $\eta$ or $\sigma$, and we denote the spin at $k$ in the configuration $\eta$ by $\eta_{k}$. Let $\mathscr{B}$ be the Borel sets of $E$ and if $F \subset Z^{d}$ let $\mathscr{B}_{F}\left(\tilde{\mathscr{B}}^{F}\right)$ denote the $\sigma$-algebra generated by $\left\{\eta_{k}: k \in F\right\}\left(\left\{\eta_{k}: k \notin F\right\}\right)$. We say a probability measure $\mu$ on $\mathscr{B}$ is a Gibbs state for the potential $\left\{J_{R}\right\}$ if a regular conditional probability distribution of $\mu$ on $\mathscr{B}_{\{k\}}$ given $\tilde{\mathscr{B}}^{\{k\}}$ is given by

$$
\varrho_{k}\left(\left\{\eta_{k}\right\} \mid \tilde{\eta}^{k}\right)=\left[1+\exp \left[2 \sum_{R \ni k} J_{R} \prod_{j \in R} \eta_{j}\right]\right]^{-1}
$$

* $\quad$ Research supported in part by N.S.F. Grant MPS74-18926. 
We are going to study mixing properties of the Gibbs states as well as the analytic dependence of their correlation functions on the potential. For example let the potential $\left\{J_{R}\right\}$ be fixed and let $\mu_{\beta}$ be a Gibbs state for the potential $\left\{\beta J_{R}\right\}$. Theorem (3.8) implies that if

(0.4) $\beta<\pi / 4 \sum_{R \ni 0}\left|J_{R}\right|$,

then $\mu_{\beta}$ is unique and for all finite $A \subset Z^{d}, \int \prod_{j \in A} \eta_{j} d \mu_{\beta}(\eta)$ can be continued analytically to the region $\left\{\beta \in \mathbb{C}:|\beta|<\Pi / \sum_{R \in 0}\left|J_{R}\right|\right\}$. As to the mixing properties, an application of Theorem (4.24) shows that if (0.4) holds and the potential has finite range, then there is an $\alpha>0$ such that for all finite $\Lambda_{0} \subset Z^{d}$ there is a constant $A\left(\Lambda_{0}\right)$ for which

$$
\sup _{B \in \mathscr{B}_{\Lambda_{0}}}\left\|\mu\left(B \mid \tilde{\mathscr{B}}^{1}\right)-\mu(B)\right\| \leqq A\left(\Lambda_{0}\right) e^{-\alpha \delta},
$$

where $\Lambda_{0} \subset \Lambda$ and $\delta$ is the distance from $\Lambda_{0}$ to the complement of $\Lambda$. The inequality $(0.5)$ of course implies that there is an exponential decay of correlations.

Both the analyticity and mixing results are true if $(0.4)$ is replaced by other conditions [see Theorem (3.10) and (4.24)]. For example, if $f \in \mathscr{C}(E)$ (the continuous functions on $E$ ) let $\|f\|$ be the supremium norm of $f$. For $k \in Z^{d}$ and $f \in \mathscr{C}(E)$ let

$$
\Delta_{k} f(\eta)=f\left({ }_{k} \eta\right)-f(\eta)
$$

where ${ }_{k} \eta$ is the configuration obtained from $\eta$ by reversing the spin at $k$. If the potential has finite range and if

$$
\sum_{k \neq 0}\left\|\Delta_{k} \varrho_{0}(\{\cdot\} \mid \cdot)\right\|<1
$$

then not only is the Gibbs state unique, but (0.5) holds.

As we mentioned the tool used to prove these theorems is the stochastic Ising model, which we now describe. Let $\mathscr{D}=\left\{f \in \mathscr{C}(E): \Delta_{k} f \equiv 0\right.$ for all but finitely many $k\}$. Let

$$
c_{k}(\eta)=2 \varrho_{k}\left(\left\{-\eta_{k}\right\} \mid \tilde{\eta}^{k}\right)
$$

and let $\mathscr{L}$ be the operator on $\mathscr{D}$ given by

$$
\mathscr{L} f(\eta)=\sum_{k \in Z^{d}} c_{k}(\eta) \Delta_{k} f(\eta)
$$

Under the condition (0.2) alone, it is not known whether $\mathscr{L}$ admits a closure which generates a strongly continuous positive contraction semigroup $\left\{T_{t}: t \geqq 0\right\}$ on $\mathscr{C}(E)$. However, if $\sum_{R \ni 0}\left|J_{R}\right|<\pi / 4$ or if $\sum_{k \neq 0}\left\|\Delta_{k} \varrho_{0}(\{\cdot\} \mid \cdot)\right\|<\infty$ holds, then not only is there one such semi-group, but there is only one (see $[5,6,3]$ and Theorems $(1,8)$ and (A.2)). Whenever there is exactly one such semi-group $\left\{T_{t}: t \geqq 0\right\}$ for a given choice of potential $\left\{J_{R}\right\}$, we call it the stochastic Ising model with potential $\left\{J_{R}\right\}$. For a description of the corresponding Markov process see [3] or [5]. If $\left\{T_{t}: t \geqq 0\right\}$ is the semi-group for the stochastic Ising model with potential $\left\{J_{R}\right\}$ and $\mu$ is a 
Gibbs state with potential $\left\{J_{R}\right\}$ then $\mu$ is $T_{t}$-stationary. That is for all $f \in \mathscr{C}(E)$ and all $t \geqq 0$

$$
\int T_{f} f(\eta) d \mu(\eta)=\int f(\eta) d \mu(\eta)
$$

(see [4]).

It is easy to understand, in general terms, why the stochastic Ising model is a powerful tool in the study of the equilibrium state. The point is that it is easier to see how the semi-group $\left\{T_{t}: t \geqq 0\right\}$ depends on the $J_{R}$ 's than it is to understand, directly, the dependence of the Gibbs states on the potential. (This circumstance is not at all surprising, since the correspondence between $\left\{J_{R}\right\}$ and $\left\{T_{t}: t \geqq 0\right\}$ is one to one far more often than that between $\left\{J_{R}\right\}$ and the Gibbs states.) If one knows, in addition, that $\left\{T_{t}: t \geqq 0\right\}$ tends to equilibrium fast enough, then one can show that the nice dependence of $\left\{T_{t}: t \geqq 0\right\}$ on $\left\{J_{R}\right\}$ is inherited by the equilibrium state. These are the basic facts of which we are going to take advantage.

In Sections 1 and 2 we prove some general facts about interacting stochastic processes. In those sections the flip rates, $c_{k}$ 's, are not required to have the form (0.8) for some potential $\left\{J_{R}\right\}$. Section 3 contains the analyticity results and Section 4 contains the mixing results. In the latter two sections we always assume that the $c_{k}$ 's are given by $(0.8)$.

\section{The Perturbation Technique}

In this section we show that the generalized stochastic Ising model [i.e. $c_{k}$ 's not required to satisfy (0.8)] can sometimes be thought of as a perturbation of the process in which each of the spins flips independently of the others.

The results in this section are a generalization of the results in Sections 6 and 7 of [3], and the reader is referred to [3] for many of the details

If $F$ is a finite subset of $Z^{d}$ and $|\alpha|<1$ let

$$
\chi_{F}^{\alpha}(\eta)=\left\{\begin{array}{lll}
1 & \text { if } & F=\emptyset \\
\prod_{j \in F}\left(\alpha+\eta_{j}\right) /(1+|\alpha|)^{|F|} & \text { if } & F \neq \emptyset .
\end{array}\right.
$$

Here $|F|$ denotes the cardinality of $F$. Note that for a given $\alpha,\left\{\chi_{F}^{\alpha}: F\right.$ finite $\}$ is the set of eigenfunctions for $\mathscr{L}^{\alpha}=\sum_{k} c_{k}^{\alpha} \Delta_{k}$, where $c_{k}^{\alpha}(\eta)=1+\alpha \eta_{k}$. Let

$$
\hat{L}_{\alpha}=\left\{f: f=\sum_{F} \hat{f}(F) \chi_{F}^{\alpha} \quad \text { where } \quad \sum_{F}|\hat{f}(F)|<\infty\right\} .
$$

For $f \in \hat{L}_{\alpha}$ we denote $\|f\|_{\alpha}=\sum_{F}|\hat{f}(F)|$. If $v^{\alpha}$ is the product measure

$$
v^{\alpha}=\prod_{j \in Z^{d}}\left(\frac{1+\alpha}{2} \delta_{\{-1\}}+\frac{1-\alpha}{2} \delta_{\{+1\}}\right)
$$

( $v^{\alpha}$ is the unique stationary distribution for $\mathscr{L}^{\alpha}$ ), then for $f \in \hat{L}_{\alpha}$ we have

$$
\hat{f}(F)=(1-|\alpha|)^{-|F|} \int \chi_{F}^{\alpha}(\eta) f(\eta) d v^{\alpha}(\eta)
$$


Thus each $f \in \hat{L}_{\alpha}$ has a unique representation in terms of the $\chi_{F}^{\alpha}$, and the series converges uniformly. In fact if $f \in \hat{L}_{\alpha}$, then

$$
\|f\| \leqq\|f\|_{\alpha} .
$$

Now consider flip rates which are of the form

$$
c_{k}(\eta)=1+\alpha \eta_{k}+\eta_{k} \sum_{G} \gamma(k, G) \chi_{G}^{\alpha}
$$

For $f \in \hat{L}_{\alpha}$ and $\lambda$ a complex number not in $\{-2 k: k=1,2, \ldots\}$ define

$$
\begin{aligned}
A_{\lambda} f= & \sum_{G} \sum_{F} \sum_{k \in Z^{d} H \subset} \sum_{H(F \backslash\{k\}) \cap G} \gamma(k, G) \frac{-2 I_{F}(k)}{\lambda+2|F|} \\
& \frac{\hat{f}(F)}{1+|\alpha|} \chi_{[(F \backslash\{k\}) \Delta G] \cup H}^{\alpha}\left(\frac{2 \alpha}{1+|\alpha|}\right)^{|H|}\left(\frac{1-|\alpha|}{1+|\alpha|}\right)^{|(F \backslash\{k\}) \cap G|-|H|}
\end{aligned}
$$

where $I_{F}(\cdot)$ is the indicator function of $F$.

(1.6) Lemma. If there is an $a \geqq 0$ such that

$$
\sum_{G}|\gamma(k, G)| \leqq a(1+|\alpha|) \quad \text { for all } k \in Z^{d},
$$

then for all complex $\lambda \neq-2,-4, \ldots$ we have

$$
\left\|A_{\lambda} f\right\|_{\alpha} \leqq a\|f\|_{\alpha} \sup _{n \geqq 1}\left|\frac{2 n}{\lambda+2 n}\right| .
$$

Proof. $\left\|A_{\lambda} f\right\|_{\alpha}$

$$
\sum_{G} \sum_{F} \sum_{k} \sum_{H \subset(F \backslash\{k\}) \cap H}|\gamma(k, G)| \frac{2 I_{F}(k)}{|\lambda+2| F||} \frac{|\hat{f}(F)|}{1+|\alpha|}\left(\frac{2|\alpha|}{1+|\alpha|}\right)^{|H|}\left(\frac{1-|\alpha|}{1+|\alpha|}\right)^{|(F \backslash\{k\}) \cap G|-|H|}
$$

Summing over $H$ first and using the equality

$$
\sum_{H \subset F \cap G}\left(\frac{2|\alpha|}{1+|\alpha|}\right)^{|H|}\left(\frac{1-|\alpha|}{1+|\alpha|}\right)^{|F \cap G|-|H|}=1
$$

and then summing over $G$ and using the hypothesis we obtain

$$
\|A f\|_{\alpha} \leqq a \sum_{F} \sum_{k} \frac{2 I_{F}(k)}{|\lambda+2| F||}|\hat{f}(F)|
$$

from which the result is obvious.

The only difference between the proofs of Lemma (7.1) and Theorem (7.10) of [3] and the proof of Theorem (1.8) below is contained in the previous lemma. (1.8) Theorem. Let $c_{k}$ be as in (1.4) and suppose that the hypotheses of Lemma (1.6) hold with $a<1$. Then there is a unique positive strongly continuous contraction semigroup $\left\{T_{t}: t \geqq 0\right\}$ on $\mathscr{C}(E)$ whose generation agrees with $\mathscr{L}=\sum_{k} c_{k} \Delta_{k}$ on $\mathscr{D}$. Moreover if for $f \in \hat{L}_{\alpha}$ we define $\Pi f$ by

$$
\Pi f=\int_{E} \sum_{n=0}^{\infty}\left(A_{0}\right)^{n} f d v^{\alpha},
$$


then there is a $\gamma>0$, depending only on a, and for each $f \in \hat{L}_{\alpha}$ a constant $D(f)$, depending only on a and $f$, such that for all $t \geqq 0$

$$
\left\|T_{t} f-\Pi f\right\| \leqq D(f) e^{-\gamma t}
$$

Proof. The proof follows almost verbatum the proofs in Section 6 and 7 of [3]. We indicate here only the necessary changes.

Let $\mathscr{L}^{\alpha}=\sum_{k}\left(1+\alpha \eta_{k}\right) \Delta_{k}$. Then since

$$
\mathscr{L}^{\alpha} \chi_{F}^{\alpha}=-2|F| \chi_{F}^{\alpha},
$$

the resolvent, $R_{\lambda}^{\alpha}$, of $\mathscr{L}^{\alpha}$ is given as follows. If $f=\sum_{F} \hat{f}(F) \chi_{F}^{\alpha} \in \hat{L}_{\alpha}$ and $\lambda \neq 0,-2$, $-4,-6, \ldots$, then

$$
R_{\lambda}^{\alpha} f=\sum_{F} \frac{1}{\lambda+2|F|} \hat{f}(F) \chi_{F}^{\alpha}
$$

Since

$$
\eta_{k} \sum_{G} \gamma(k, G) \chi_{G}^{\alpha}(\eta) \Delta_{k} \chi_{F}^{\alpha}(\eta)=-2 \sum_{G} \frac{\gamma(k, G)}{1+|\alpha|} I_{F}(k) \chi_{G}^{\alpha}(\eta) \chi_{F \backslash\{k\}}^{\alpha}(\eta),
$$

and

$$
\chi_{F}^{\alpha} \chi_{G}^{\alpha}=\sum_{H \subset F \cap G} \chi_{(F \Delta G) \cup H}^{\alpha}\left(\frac{2 \alpha}{1+|\alpha|}\right)^{|H|}\left(\frac{1-|\alpha|}{1+|\alpha|}\right)^{|F \cap G|-|H|},
$$

we see that for $f \in \hat{L}_{\alpha}$

$$
\left(\mathscr{L}-\mathscr{L}^{\alpha}\right) R_{\lambda}^{\alpha} f=A_{\lambda} f .
$$

The proof now follows exactly along the lines of [3].

(1.11) Remark. (1.10) implies that the semi-group has a unique stationary measure, $\mu$, and (1.9) implies that for $f \in \hat{L}_{\alpha}$

$$
\int f d \mu=\int \sum_{n=0}^{\infty}\left(A_{0}\right)^{n} f d \nu^{\alpha}
$$

\section{The Theorems of Dobrushin and Sullivan}

In this section we prove a theorem which is related to a theorem of Dobrushin [1] and is a particular case of a theorem due to Sullivan [6]. The reason for including another proof here is that it can be greatly simplified in the case which we are considering.

Recall that $\Delta_{k} f$ is given by (0.6). Since the operators $\Delta_{k}$ are analogous to partial derivatives with respect to the $k^{\text {th }}$ variable, we will often write $f_{, k}$ for $\Delta_{k} f$. For $f \in \mathscr{C}(E)$ let

$$
\|f\|=\sum_{k}\left\|f_{, k}\right\| \leqq \infty
$$


Let $\mathscr{C}^{1}(E)=\{f \in \mathscr{C}(E):\|f\| \mid<\infty\}$. It is known (see [3]) that if

$$
\sup _{k}\left[\left\|c_{k}\right\|+\left\|c_{k}\right\|\right]=C<\infty,
$$

then there is exactly one positive, strongly continuous contraction semigroup $\left\{T_{t}: t \geqq 0\right\}$ on $\mathscr{C}(E)$ whose generator agrees with $\mathscr{L}=\sum_{k} c_{k} \Delta_{k}$ on $\mathscr{D}$. Moreover, if we denote the generator of $T_{t}$ by $\overline{\mathscr{L}}$, then the domain of $\overline{\mathscr{L}}$ includes $\mathscr{C}^{1}(E)$; and if $f \in \mathscr{C}(E)$,

(2.3) $\quad\left\|T_{t} f\right\| \leqq e^{C t}\|f\| \|$.

(2.4) Theorem (Dobrushin-Sullivan). Let $\left\{c_{k}: k \in Z^{d}\right\} \subset C^{+}(E)$ satisfy (2.2). Let

$$
\gamma=\inf _{k} \inf _{\eta}\left(c_{k}(\eta)+c_{k}\left({ }_{k} \eta\right)\right)-\sup _{k} \sum_{j \neq k}\left\|c_{k, j}\right\|
$$

Set $\mathscr{L}=\sum_{k} c_{k} \Delta_{k}$ on $\mathscr{D}$ and let $\left\{T_{t}: t \geqq 0\right\}$ be the associated Feller semi-group on $\mathscr{C}(E)$. Then

$\left\|T_{t} \varphi\right\| \leqq e^{-\gamma t}\|\varphi\| \|, \quad t \geqq 0 \quad$ and $\quad \varphi \in \mathscr{C}(E)$.

In particular, if $\gamma>0$, then there is a unique probability measure $\mu$ on $E$ such that

$$
\left\|T_{t} \varphi-\int \varphi d \mu\right\| \leqq M e^{-\gamma t} \mid\|\varphi\| \|, \quad t \geqq 0
$$

where $M=\sup _{k}\left\|c_{k}\right\|$.

Proof. From (4.11) in [3], we know that (2.3) holds. Thus if $\lambda>C$ and $f=R_{\lambda} \varphi$ for some $\varphi \in \mathscr{C}^{1}(E)$, then $\|f\|\|\leqq\| \varphi \| /(\lambda-C)$ and

$$
\lambda f-\sum_{j} c_{j} f_{, j}=\varphi
$$

Thus

$$
\lambda f_{, k}(\eta)=\varphi_{, k}+\sum_{j \neq k} c_{j}\left({ }_{k} \eta\right) f_{, j, k}(\eta)+\sum_{j \neq k} c_{j, k}(\eta) f_{, j}(\eta)+\left(c_{k} f_{, k}\right)_{, k}
$$

Since $E$ is compact and $f_{, k}\left({ }_{k} \eta\right)=-f_{, k}(\eta)$, we can find $\eta^{*} \in E$ such that

$$
f_{, k}\left(\eta^{*}\right)=\max _{\eta}\left|f_{, k}(\eta)\right|
$$

Note that $f_{, k, j}\left(\eta^{*}\right) \leqq 0$ for all $j$ and

$$
\begin{aligned}
\left(c_{k} f_{, k}\right)_{, k}\left(\eta^{*}\right) & =c_{k, k}\left(\eta^{*}\right) f_{, k}\left({ }_{k} \eta^{*}\right)+c_{k}\left(\eta^{*}\right) f_{, k, k}\left(\eta^{*}\right) \\
& =-f_{, k}\left(\eta^{*}\right)\left(c_{k, k}\left(\eta^{*}\right)+2 c_{k}\left(\eta^{*}\right)\right) \\
& =-\left(c_{k}\left(\eta^{*}\right)+c_{k}\left({ }_{k} \eta^{*}\right)\right)\left\|f_{, k}\right\| .
\end{aligned}
$$

Hence

$$
\lambda\left\|f_{, k}\right\| \leqq\left\|\varphi_{, k}\right\|-\left(c_{k}\left(\eta^{*}\right)+c_{k}\left({ }_{k} \eta^{*}\right)\right)\left\|f_{, k}\right\|+\sum_{j \neq k}\left\|c_{j, k}\right\|\left\|f_{, j}\right\|
$$


and so

$$
\begin{aligned}
& \lambda\|f\| \leqq\|\varphi\|-\inf _{k, \eta}\left(c_{k}(\eta)+c_{k}\left({ }_{k} \eta\right)\right)\|\| f\|\| \\
& +\sum_{j} \sum_{k \neq j}\left\|c_{j, k}\right\|\left\|f_{, j}\right\| \\
& \leqq\|\varphi\|-\gamma\|f\| \| .
\end{aligned}
$$

Thus if $\lambda>\max (-\gamma, C)$, then $\left\|R_{\lambda} \varphi\right\|\|\leqq\| \varphi \| /(\lambda+\gamma)$. Using the well-known formula

$$
T_{t} \varphi=\lim _{\lambda \rightarrow \infty} e^{-\lambda t} \sum_{n=0}^{\infty}\left(\lambda^{2} t R_{\lambda}\right)^{n} \varphi / n !
$$

one easily gets

$$
\left\|T_{t} \varphi\right\|\left\|e^{-\gamma t}\right\| \varphi\|\|
$$

Finally, if $\gamma>0$ and $\varphi \in \mathscr{C}^{1}(E)$, then

$$
\frac{\partial T_{t} \varphi}{\partial t}=\mathscr{L} T_{t} \varphi
$$

and so

$$
\left\|\frac{\partial T_{t} \varphi}{\partial t}\right\| \leqq M\left\|T_{t} \varphi\right\| \geqq M e^{-\gamma t}\|\varphi\| .
$$

Hence

$$
\left\|T_{t} \varphi-T_{s} \varphi\right\| \leqq M e^{-\gamma s}\|\varphi\| \text { for } t \geqq s .
$$

The last part of the theorem follows immediately from this.

\section{Analyticity of the Correlation Functions}

Let $U$ be a connected open set in the complex plane and let $\left\{J_{R}(z): R\right.$ a finite subset of $\left.Z^{d}\right\}$ be a collection of analytic functions which satisfy $(0.1)$ and $(0.2)$. Assume also that there is at least one real $z \in U$ and that $J_{R}(z)$ is real for real $z \in U$. Then for real $z \in U$ there is at least one Gibbs state, $\mu_{z}$, corresponding to the potential $\left\{J_{R}(z)\right\}$, and we know that whenever one of the perturbation techniques of section one can be applied there is exactly one such Gibbs state. The goal of this section is to obtain conditions under which, for all $f \in \mathscr{D}, \int f d \mu_{z}$ can be continued analytically to an open set containing the intersection of $U$ and the real axis.

(3.1) Lemma. Let $U$ be a connected open subset of $\mathbb{C}$ containing at least one real number. For $k \in Z^{d}$ and finite $G \subset Z^{d}$, let $\gamma(\cdot, k, G)$ be a function on $U$ with the property that for some $\alpha \in(-1,1)$ and $a \in[0,1)$

$$
c_{k}(z, \eta)=1+\alpha \eta_{k}+\eta_{k} \sum_{G} \gamma(z, k, G) \chi_{G}^{\alpha}(\eta)
$$

is non-negative for real $z \in U$ and

(3.3) $\sup _{z \in U} \sup _{k} \sum_{G}|\gamma(z ; k, G)| \leqq a(1+|\alpha|)$. 
If $z \in U$ is real let $T_{t}^{(z)}$ be the Feller semi-group determined by $\mathscr{L}^{(z)}=\sum_{k} c_{k}(z, \cdot) \Delta_{k}$. By Theorem (1.8) there is, for each real $z \in U$, a unique probability measure, $\mu_{z}$, such that

$$
\int T_{t} f d \mu_{z}=\int f d \mu_{z} \text { for all } f \in \mathscr{C}(E) .
$$

If in addition to (3.3) we assume that

(3.4) $\gamma(z ; k, G)$ is an analytic function of $z \in U$ for all $k \in Z^{d}$ and $G \subset Z^{d}$ and for each $k$, $\sum_{G}|\gamma(z ; k, G)|$ converges uniformly in $z \in U$,

then for all $f \in \mathscr{D}, \int f d \mu_{z}$ may be continued analytically to $U$.

Proof. Since $\mathscr{D} \subset \hat{L}_{\alpha}$, it follows from Theorem (1.8) that if $f \in \mathscr{D}$ and $z \in U$ is real then

(3.5) $\int f d \mu_{z}=\int \sum_{n=0}^{\infty}\left(A_{0}^{(z)}\right)^{n} f d v^{\alpha}$,

where $A_{0}^{(z)}$ is defined by (1.5) using $\gamma(z ; k, G)$. But from (3.4) it is easily checked that the right side of (3.5) is an analytic function of $z \in U$.

(3.6) Theorem. Let $U$ and $\left\{J_{R}(z)\right\}$ be as in the first paragraph of this section. Assume that there is an $\alpha \in(-1,1)$ and an $a \in[0,1)$ such that if $\gamma(z ; k, G)$ is given by

$$
\gamma(z: k, G)=(1-|\alpha|)^{-|G|} \int \chi_{G}^{\alpha}(\eta)\left[\tanh \left(\sum_{R \ni k} J_{R}(\eta) \prod_{j \in R \backslash\{k\}} \eta_{j}\right)-\alpha\right] d v^{\alpha}(\eta)
$$

then $\gamma(z ; k, G)$ satisfies (3.3) and (3.4). Then for all real $z \in U$ there is a unique Gibbs state, $\mu_{z}$, with potential $\left\{J_{R}(z)\right\}$, and if $f \in \mathscr{D}, \int f d \mu_{z}$ can be continued analytically to $U$.

Proof. For $z \in U$ let

$$
\begin{aligned}
c_{k}(z, \eta) & =2\left(1+\exp \left[2 \sum_{R \ni k} J_{R}(z) \prod_{j \in R} \eta_{j}\right]\right)^{-1} \\
& =1+\alpha \eta_{k}+\eta_{k}\left[\tanh \left(\sum_{R \ni k} J_{R}(z) \prod_{j \in R \backslash\{k\}} \eta_{j}\right)-\alpha\right] .
\end{aligned}
$$

If $z \in U$ is real let $\mu_{z}$ be the stationary measure for the semi-group determined by $\sum_{k} c_{k}(z, \cdot) \Delta_{k}$. Then by Theorem (1.8) and (0.10), $\mu_{z}$ is the unique Gibbs state for the potential $\left\{J_{R}(z)\right\}$. The rest now follows from Lemma (3.1).

(3.8) Theorem. Let $U$ and $\left\{J_{R}(z)\right\}$ be as in the first paragraph of this section. Assume that $\sum_{R \ni 0}\left|J_{R}(z)\right|$ converges uniformly for $z \in U$ and that

$$
\sup _{z \in U} \sum_{R \ni 0}\left|J_{R}(z)\right|<\pi / 4
$$

Then for each real $z \in U$ there is a unique Gibbs state, $\mu_{z}$, with potential $\left\{J_{R}(z)\right\}$ and for each $f \in \mathscr{D}, \int f d \mu_{z}$ can be continued analytically to $U$.

Proof. We take $\alpha=0$ in (3.7) and check that there is an $a \in[0,1)$ such that (3.3) and (3.4) hold. The analyticity of each $\gamma(z ; k, G)$ follows from the dominated con- 
vergence theorem since for each $\eta, \sum_{R \ni k} J_{R}(z) \prod_{j \in R \backslash\{k\}} \eta_{j}$ is an analytic function which is bounded by $\pi / 4$.

The verification of (3.3) and the uniformity statement in (3.4) is deferred to Theorem (A.2) of the Appendix.

(3.10) Theorem. Let $U$ and $\left\{J_{R}(z)\right\}$ be given as in the preceding, and assume, in addition, that

(i) the series

(3.11) $\sum_{R \ni 0}\left|J_{R}(z)\right| 3^{|R|}$

converges uniformly for $z \in U$,

(ii) $J_{\{0\}}(z) \neq 0$ for any $z \in U$ and

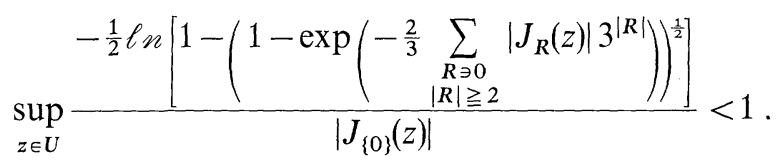

Then for each real $z \in U$ there is a unique Gibbs state $\mu_{z}$. Moreover, there is an open set $V \leqq U$ such that $U \cap\{$ reals $\} \subseteq V$ and for all $f \in \mathscr{D}$ the map $z \rightarrow \int f d \mu_{z}$, $z \in U \cap\{$ reals $\}$, admits an analytic continuation to $V$.

The proof of Theorem (3.10) is deferred to Theorem (A.14) in the appendix, where it is shown that each real $z_{0} \in U$ is contained in an open set $U_{z_{0}} \subset U$ on which (3.3) and (3.4) hold.

\section{A Strong Cluster Property}

Let $\left\{J_{R}\right\}$ be a potential such that there is only one Gibbs state, $\mu$, corresponding to $\left\{J_{R}\right\}$. Dobrushin has shown (see [2]) that this is equivalent to the condition that for all $f \in \mathscr{D}$ and all sequences $\left\{\Lambda_{n}\right\}$ of finite subsets of $Z^{d}$ such that $\Lambda_{n} \cong \Lambda_{n+1}$ and $\bigcup_{n} \Lambda_{n}=Z^{d}$

(4.1) $\lim _{n \rightarrow \infty}\left\|E^{\mu}\left[f \mid \tilde{\mathscr{B}}^{1_{n}}\right]-E^{\mu}[f]\right\|=0$.

Our goal in this section is to find conditions on the potential $\left\{J_{R}\right\}$ which guarantee that the convergence in (4.1) is exponentially fast.

The technique is to represent $E^{\mu}\left[f \mid \tilde{\mathscr{B}}^{1_{n}}\right](\xi)$ as the expectation of $f$ with respect to the stationary measure of the semi-group generated by the bounded operator $\mathscr{L}^{\Lambda_{n}, \xi}$ given by

$$
\mathscr{L}^{\Lambda_{n}, \xi} f(\eta)=\sum_{k \in \Lambda_{n}} c_{k}\left(\sigma\left(\Lambda_{n}, \xi, \eta\right)\right) \Delta_{k} f(\eta),
$$

where $\sigma\left(\Lambda_{n}, \xi, \eta\right)$ is the element of $E$ given by

$$
\sigma\left(\Lambda_{n}, \xi, \eta\right)_{k}=\left\{\begin{array}{lll}
\eta_{k} & \text { if } & k \in \Lambda_{n} \\
\xi_{k} & \text { if } & k \notin \Lambda_{n} .
\end{array}\right.
$$


If $\mu^{\Lambda_{n}, \xi}(\cdot)$ is the probability measure on $\{-1,1\}^{\Lambda_{n}}$ with

$$
\mu^{\Lambda_{n}, \xi}(\{\eta\})=\left[Z\left(\Lambda_{n}, \xi\right)\right]^{-1} \exp \left[-\sum_{R \wedge A_{n} \neq \emptyset} J_{R} \prod_{j \in R} \sigma\left(\Lambda_{n}, \xi, \eta\right)_{j}\right],
$$

where $Z\left(\Lambda_{n}, \xi\right)$ is the normalizing constant, then for all $f \in \mathscr{C}\left(\{-1,1\}^{\Lambda_{n}}\right.$

(4.4) $\int f d \mu^{\Lambda_{n}, \xi}=E^{\mu}\left[f \mid \tilde{\mathscr{B}}^{\Lambda_{n}}\right](\xi)$,

and

(4.5) $\int \mathscr{L}^{\Lambda_{n}, \xi} f(\eta) d \mu^{\Lambda_{n}, \xi}(\eta)=0$.

Since $\mathscr{L}^{\Lambda_{n}, \xi}$ is a bounded operator, there is no doubt that it generates a unique semi-group $\left\{T_{t}^{\Lambda_{n}, \xi}: t \geqq 0\right\}$ and that

$$
\int f d \mu^{A_{n}, \xi}=\int T_{t}^{1_{n}, \xi} f d \mu^{A_{n}, \xi}
$$

for all $f \in \mathscr{C}(E)$.

(4.7) Theorem. Suppose $\alpha$ is a positive number with the property that for all $\Lambda$, $\xi \in E$, and $f \in \mathscr{D}(\Lambda) \equiv\left\{f \in \mathscr{D}: \Delta_{k} f \equiv 0\right.$ for $\left.k \notin \Lambda\right\}$, there is an $A(f)<\infty$, not depending on $\Lambda$, such that:

$$
\left\|T_{t}^{1, \xi} f-\int f d \mu^{1, \xi}\right\| \leqq A(f) e^{-\alpha t}, \quad t \geqq 0 .
$$

Then $\left\{T_{t}: t \geqq 0\right\}$ admits exactly one stationary distribution $\mu$ and for $f \in \mathscr{D}$ :

$$
\left\|T_{t} f-\int f d \mu\right\| \leqq A(f) e^{-\alpha t}, \quad t \geqq 0 .
$$

Assume in addition that there is an $M<\infty$ such that

$$
\Delta_{j} c_{k} \equiv 0 \text { if }|j-k| \geqq M,
$$

or equivalently, that

(4.11) $J_{R}=0$ if $0 \in R \nsubseteq[-M, M]^{d}$.

Then if $\Lambda \subset \tilde{\Lambda}$ and $\varrho$ is the distance between $\Lambda$ and the complement of $\tilde{\Lambda}$, one has for $f \in \mathscr{D}(\Lambda)$ and $\xi \in E$ :

$$
\left|\int f d \mu-\int f d \mu^{\tilde{1}, \xi}\right| \leqq 2(A(f)+\|f\| \mid) e^{-\alpha \gamma \varrho / c M},
$$

where $C=\max \left(\left\|c_{0}\right\|,\left\|c_{0}\right\|\right)$ and $\gamma \in(0,1)$ solves

$$
(1+\alpha / C) \gamma+\ell n \gamma+1=0 \text {. }
$$

Proof. That (4.8) implies (4.9) is an easy consequence of the fact that $T_{t}^{\Lambda_{n}, \xi} f \rightarrow T_{t} f$ as $\Lambda_{n} \nearrow Z^{d}$ (see [3] or [5]). To prove the second assertion, note that:

$$
\begin{aligned}
& \left|\int f d \mu-\int f d \mu^{\tilde{\Lambda}, \xi}\right| \\
& \leqq\left|\int f d \mu-T_{t} f(\eta)\right|+\left|T_{t} f(\eta)-T_{t}^{\widetilde{\Lambda}, \xi} f(\eta)\right|+\left|T^{\widetilde{\Lambda}, \xi} f(\eta)-\int f d \mu^{\widetilde{\Lambda}, \xi}\right| \\
& \leqq 2 A(f) e^{-\alpha t}+\left|T_{t} f(\eta)-T_{t}^{\widetilde{\Lambda}, \xi} f(\eta)\right|
\end{aligned}
$$

To bound $\left|T_{t} f(\eta)-T_{t}^{\tilde{\Lambda}, \xi} f(\eta)\right|$ we observe that

$$
T_{0} f(\cdot)-T_{0}^{\tilde{\Lambda}, \xi} f(\cdot)=0
$$


and

$$
\begin{aligned}
\frac{\partial}{\partial t}\left(T_{t} f(\cdot)-T_{t}^{\tilde{\Lambda}, \xi} f(\cdot)\right) & =\mathscr{L} T_{t} f-\mathscr{L}_{t}^{\tilde{\Lambda}, \xi} T_{t}^{\tilde{\Lambda}, \xi} f \\
& =\mathscr{L}^{\tilde{\Lambda}, \xi}\left(T_{t} f-T_{t}^{\tilde{\Lambda}, \xi} f\right)+\left(\mathscr{L}-\mathscr{L}^{\tilde{\Lambda}, \xi}\right) T_{t} f
\end{aligned}
$$

Thus

$$
T_{t} f-T_{t}^{\tilde{\Lambda}, \xi} f=\int_{0}^{t} T_{t-s}^{\tilde{\Lambda}, \xi}\left(\mathscr{L}-\mathscr{L}^{\tilde{\Lambda}, \xi}\right) T_{s} f d s
$$

and

$$
\left|T_{t} f(\eta)-T_{t}^{\tilde{\Lambda}, \xi} f(\eta)\right| \leqq 2 \int_{0}^{t} \sum_{k \notin \Lambda_{0}}\left\|c_{k}\right\|\left\|\Delta_{k} T_{s} f\right\| d s,
$$

where $\Lambda_{0}=\left\{j \in Z^{d}=\right.$ the distance from $j$ to the complement of $\tilde{\Lambda}$ is at least $\left.M\right\}$. We need the following lemma.

(4.15) Lemma. Assume that (4.10) holds and let $f \in \mathscr{D}(\Lambda)$ and $C$ be as in (4.12). Set $\Lambda^{(N)}=\left\{k \in Z^{d}: \operatorname{dist}(k, \Lambda) \leqq N M\right\}$. Then

$$
\sum_{k \notin \Lambda(N)}\left\|\Delta_{k} T_{s} f\right\| \leqq\left(c^{C s}-\sum_{j=0}^{N} \frac{(C s)^{j}}{j !}\right)\|f\| \|
$$

We postpone the proof of the lemma and complete the proof of the theorem.

Let $[\varrho / M]$ be the integral part of $\varrho / M$ and set $N=[\varrho / M]-1$. Then $\Lambda_{0} \supset \Lambda^{(N)}$ and the right side of (4.14) is bounded by

$$
\begin{aligned}
2 C \int_{0}^{t} \sum_{k \notin \Lambda^{(N)}}\left\|\Delta_{k} T_{s} f\right\| d s & \leqq 2 C \int_{0}^{t}\left(e^{C s}-\sum_{j=0}^{N} \frac{(C s)^{j}}{j !}\right) d s\|f\| \\
& \leqq 2 e^{C t} \frac{(C t)^{N+2}}{(N+2) !}\|f\|
\end{aligned}
$$

Combining (4.13), (4.14), and (4.17) we have

$$
\left|\int f d \mu-\int f d \mu^{\tilde{A}, \xi}\right| \leqq 2 A(f) e^{-\alpha t}+2\|f\| \| e^{C t} \frac{(C t)^{[\varrho / M]+1}}{([\varrho / M]+1) !}
$$

Setting $t=\gamma([\varrho / M]+1) / C$ and using the bounds $k ! \geqq k^{k} e^{-k}$ and $[\varrho / M]+1 \geqq \varrho / M$, we get the desired conclusion.

Proof of Lemma (4.15). Let $f$ be as in the statement of the lemma and note that

$$
\frac{\partial}{\partial t} \Delta_{j} T_{t} f(\eta)=\mathscr{L} \Delta_{j} T_{t} f(\eta)+\sum_{k}\left(\Delta_{j} c_{k}(\eta)\right) \Delta_{k} T_{t} f\left({ }_{j} \eta\right)
$$

and

(4.20) $\Delta_{j} T_{0} f(\eta)=\Delta_{j} f(\eta)$. 
The solution of (4.19) with initial data (4.20) is

$$
\left.\Delta_{j} T_{t} f(\eta)=T_{t} \Delta_{j} f(\eta)+\int_{0}^{t} T_{t-s} \sum_{k}\left(\Delta_{j} c_{k}(\eta)\right) \Delta_{k} T_{s} f\left({ }_{j} \eta\right)\right) d s
$$

Note that if $j \notin \Lambda$ we have $\Delta_{j} f \equiv 0$ and hence

$$
\left\|\Delta_{j} T_{t} f(\cdot)\right\| \leqq \int_{0}^{t} \sum_{k}\left\|\Delta_{j} c_{k}(\cdot)\right\|\left\|\Delta_{k} T_{s} f(\cdot)\right\| d s
$$

Thus

$$
\begin{aligned}
\sum_{j \notin \Lambda^{(N)}}\left\|\Delta_{j} T_{t} f(\cdot)\right\| & \leqq \int_{0}^{t} \sum_{k} \sum_{j \notin \Lambda^{(N)}}\left\|\Delta_{j} c_{k}(\cdot)\right\|\left\|\Delta_{k} T_{s} f(\cdot)\right\| d s \\
& \leqq \int_{0}^{t} \sum_{k \notin \Lambda^{(N-1)}} \sum_{j}\left\|\Delta_{j} c_{k}(\cdot)\right\|\left\|\Delta_{k} T_{s} f(\cdot)\right\| d s .
\end{aligned}
$$

Since $\sum_{j}\left\|\Delta_{j} c_{k}\right\| \leqq C$ we have

$$
\sum_{j \notin \Lambda^{(N)}}\left\|\Delta_{j} T_{t} f(\cdot)\right\| \leqq C \int_{0}^{t} \sum_{j \notin \Lambda^{(N-1)}}\left\|\Delta_{j} T_{s} f(\cdot)\right\| d s .
$$

The proof is now completed by induction, beginning with (2.3).

(4.24) Theorem. Let $\left\{J_{R}\right\}$ be a potential satisfying (0.1), (4.11), and one of the following:

a) $\sum_{k=0}\left\|\Delta_{k}\left(1+\exp \left[2 \sum_{R \ni 0} J_{R} \chi_{R}^{0}\right]\right)^{-1}\right\|<1$

b) $\sum_{R \ni 0}\left|J_{R}\right|<\pi / 4$

c) $\left|J_{\{0\}}\right|>-\frac{1}{2} \ln \left(1-\left[1-\exp \left(-\frac{2}{3} \sum_{\substack{R \ni 0 \\|R| \geqq 2}}\left|J_{R}\right| 3^{|R|}\right)\right]^{\frac{1}{2}}\right)$.

Then there is exactly one Gibbs state $\mu$ with potential $\left\{J_{R}\right\}$. Moreover, there is a $\gamma>0$ such that for each $f \in \mathscr{D}$ there exists a constant $A(f)<\infty$ with the property that if $f \in \mathscr{D}(\Lambda)$ then

$$
\left\|E^{\mu}\left[f \mid \tilde{B}^{\tilde{\Lambda}}\right]-E^{\mu}[f]\right\| \leqq A(f) e^{-\gamma \varrho},
$$

where $\tilde{\Lambda} \supset \Lambda$ and $\varrho$ is the distance from $\Lambda$ to the complement of $\tilde{\Lambda}$.

Proof. We need only check the hypotheses of Theorem (4.7). To do this, note that Theorems (2.4) and (1.8) apply to finite as well as infinite systems, and the conclusions of Theorems (2.4) and (1.8) imply the hypotheses of Theorem (4.7). Thus we need only check the hypotheses of Theorems (2.4) and (1.8). The inequality a) is easily seen to imply the hypotheses of Theorem (2.4) and Theorems (A.2) and (A.14) in the appendix show that Theorem (1.8) is applicable under conditions b) and $\mathrm{c})$.

(4.25) Remark. Dobrushin [2] has proved uniqueness of the Gibbs state under condition a) of Theorem (4.24), but the other conclusion of Theorem (4.24) does not follow merely from uniqueness of the Gibbs state and finiteness of the range of the potential. The two dimensional nearest neighbor ferromagnetic potential at the critical temperature provides a counter-example. 


\section{Appendix}

For $-1<\alpha<1$ let $\chi_{F}^{\alpha}$ and $\hat{L}_{\alpha}$ be as in section one. For $\alpha \geqq 1$ let

$$
\hat{L}_{\alpha}=\left\{f \in \hat{L}_{0}: \sum_{Z}|\hat{f}(F)| \alpha^{|F|} \equiv\|f\|_{\alpha}<\infty\right\} .
$$

(A.1) Lemma. $L_{\alpha}$ is a Banach algebra for all $\alpha>-1$.

Proof. That $\hat{L}_{\alpha}$ is a Banach space is obvious. Thus we need only check that $\|f \cdot g\|_{\alpha} \leqq\|f\|_{\alpha}\|g\|_{\alpha}$. Consider first the case $|\alpha|<1$. Let $f, g \in \hat{L}_{\alpha}, f=\sum_{F} \hat{f}(F) \chi_{F}^{\alpha}$ and $g=\sum_{F} \hat{g}(F) \chi_{F}^{\alpha}$. Then

$$
\begin{aligned}
f \cdot g & =\sum_{F} \sum_{G} \hat{f}(F) \hat{g}(G) \chi_{F}^{\alpha} \chi_{G}^{\alpha} \\
& =\sum_{F} \sum_{G} \sum_{H \subset F \cap G} \hat{f}(F) \hat{g}(G) \chi_{(F \Delta G) \cup H}^{\alpha}\left(\frac{2 \alpha}{1+|\alpha|}\right)^{|H|}\left(\frac{1-|\alpha|}{1+|\alpha|}\right)^{|F \cap G|-|H|}
\end{aligned}
$$

Therefore

$$
\begin{aligned}
\|f \cdot g\|_{\alpha} & \leqq \sum_{F} \sum_{G} \sum_{H \subset F \cap G}|\hat{f}(F)||\hat{g}(G)|\left(\frac{2|\alpha|}{1+|\alpha|}\right)^{|H|}\left(\frac{1-|\alpha|}{1+|\alpha|}\right)^{|F \cap G|-|H|} \\
& =\sum_{F}|\hat{f}(F)| \sum_{G}|\hat{g}(G)|=\|f\|_{\alpha}\|g\|_{\alpha} .
\end{aligned}
$$

Now consider $\alpha \geqq 1$.

$$
f \cdot g=\sum_{F} \sum_{G} \hat{f}(F) \hat{g}(G) \chi_{F \Delta G}^{0} \cdot
$$

Thus

$$
\begin{aligned}
\|f \cdot g\|_{\alpha} & \leqq \sum_{F} \sum_{G}|\hat{f}(F)||\hat{g}(G)| \alpha^{|F \Delta G|} \\
& \leqq \sum_{F} \sum_{G}|\hat{f}(F)||\hat{g}(G)| \alpha^{|F|+|G|}=\|f\|_{\alpha}\|g\|_{\alpha} .
\end{aligned}
$$

(A.2) Theorem. Under the hypotheses of Theorem (3.8) both (3.3) with $\alpha=0$ and (3.4) hold.

Proof. Let $a=\tan \sup _{z \in U} \sum_{R \ni 0}\left|J_{R}(z)\right|$. Because of (3.9), $a<1$.

Now if $|\omega|<\pi / 2$ then

(A.3) $\tanh (\omega)=\sum_{n=1}^{\infty} 2^{2 n}\left(2^{2 n}-1\right) B_{2 n} \omega^{2 n-1} /(2 n)$ !

and

$$
\tan (\omega)=\sum_{n=1}^{\infty} 2^{2 n}\left(2^{2 n}-1\right)\left|B_{2 n}\right| \omega^{2 n-1} /(2 n) !,
$$

where the $B_{2 n}$ 's are the Bernoulli numbers. Thus it follows from Lemma (A.1) applied to $\hat{L}_{0}$, and the bound

$$
\left\|\sum_{R \ni 0} J_{R}(z) \chi_{R \backslash\{0\}}^{0}\right\|_{0} \leqq \arctan (a)
$$


that for all $z \in U$

$$
\left\|\tanh \left(\sum_{R \ni 0} J_{R}(z) \chi_{R \backslash\{0\}}^{0}\right)\right\|_{0} \leqq a
$$

i.e. that (3.3) holds. With regards to (3.4), we have already seen in the proof of Theorem (3.8) that each $\gamma(z ; k, G)$ is analytic in $z \in U$. The uniform convergence of $\sum_{G}|\gamma(z ; k, G)|$ follows easily from Lemma (A.1) and Equation (A.3), since we are assuming that $\sum_{R \ni 0}\left|J_{R}(z)\right|$ converges uniformly for $z \in U$.

(A.4) Lemma. Let $|\alpha|<1$ and $f \in \hat{L}_{1+2|\alpha|}$. Then $f \in \hat{L}_{\alpha}$ and $\|f\|_{\alpha} \leqq\|f\|_{1+2|\alpha|}$.

Proof.

$$
\begin{aligned}
\chi_{F}^{0}(\eta) & =\prod_{j \in F}\left(\alpha+\eta_{j}-\alpha\right)=\sum_{H \subset F} \prod_{j \in H}\left(\alpha+\eta_{j}\right)(-\alpha)^{|F|-|H|} \\
& =\sum_{H \subset F} \chi_{H}^{\alpha}(1+|\alpha|)^{|H|}(-\alpha)^{|F|-|H|}
\end{aligned}
$$

Thus

$$
f=\sum_{F} \hat{f}(F) \chi_{F}^{0}=\sum_{F} \sum_{H \subset F} \hat{f}(F)(1+|\alpha|)^{|H|}(-\alpha)^{|F|-|H|} \chi_{H}^{\alpha} .
$$

Both conclusions of the lemma follow now from

$$
\begin{aligned}
\|f\|_{\alpha} & \leqq \sum_{F} \sum_{H \subset F}|\hat{f}(F)|(1+|\alpha|)^{|H|}|\alpha|^{|F|-|H|} \\
& =\sum_{F}|\hat{f}(F)|(1+2|\alpha|)^{|F|}=\|f\|_{1+2|\alpha|} .
\end{aligned}
$$

(A.5) Lemma. If $h>|v|$ then

(A.6) $\tanh (v+h)=1+2 \sum_{k=1}^{\infty}(-1)^{k} e^{-2 k(v+h)}$.

Proof. Since $h>|v|$ the summation on the right side of (A.6) converges. The proof is accomplished by performing the summation and using the definition of tanh.

(A.7) Lemma. If $V(\eta)=\sum_{R \ni 0} J_{R} \chi_{R \backslash\{0\}}^{0}(\eta) \in \hat{L}_{3}$ and $H>\|V\|_{3}$ then there is an $\alpha \in(0,1)$ such that

(A.8) $\tanh (V(\eta)+H)=\alpha+\sum_{G} \gamma(G) \chi_{G}^{\alpha}(\eta)$

and

(A.9) $\sum_{G}|\gamma(G)| \leqq 2 \sum_{k=1}^{\infty} e^{-2 k H}\left(e^{2 k\|V\|_{1}+2|\alpha|}-1\right)$.

Proof. Since $|V(\eta)| \leqq\|V\|_{3}<H$, it follows that $1>\tanh (V(\eta)+H)>0$ for all $\eta$. Letting $v^{\alpha}$ be as in (1.1) we have

$$
\int \tanh (V(\eta)+H) d v^{\alpha}(\eta)-\alpha
$$


is positive for $\alpha=0$, negative for $\alpha=1$ and continuous as a function of $\alpha$. Thus there is an $\bar{\alpha} \in(0,1)$ such that

(A.10) $\int \tanh (V(\eta)+H) d \nu^{\bar{\alpha}}(\eta)-\bar{\alpha}=0$.

Now using Lemma (A.5) we have

(A.11) $\tanh (V(\eta)+H)$

$$
=\bar{\alpha}+\left(1-\bar{\alpha}+2 \sum_{k=1}^{\infty}(-1)^{k} e^{-2 k H}+2 \sum_{k=1}^{\infty}(-1)^{k} e^{-2 k H}\left(e^{-2 k V(\eta)}-1\right)\right) .
$$

Lemmas (A.1) and (A.4) imply that

(A.12) $1-\bar{\alpha}-\frac{2 \mathrm{e}^{-2 H}}{1+e^{-2 H}}+2 \sum_{k=1}^{\infty}(-1)^{-2 k H}\left(e^{-2 k V(\eta)}-1\right) \in \hat{L}_{\bar{\alpha}}$.

Also the integral of (A.12) with respect to $v^{\bar{\alpha}}$ is zero. Hence when (A.12) is expanded in terms of $\chi_{F}^{\bar{\alpha}}$, s the constant term is zero. Thus

$$
\begin{aligned}
& \left\|1-\bar{\alpha}-\frac{2 e^{-2 H}}{1+e^{-2 H}}+2 \sum_{k=1}^{\infty}(-1)^{k} e^{-2 k H}\left(e^{-2 k V(\cdot)}-1\right)\right\|_{\bar{\alpha}} \\
& =2\left\|\sum_{k=1}^{\infty}(-1)^{k} e^{-2 k H}\left(e^{-2 k V(\cdot)}-1\right)\right\|_{\bar{\alpha}}-\left|1-\bar{\alpha}-\frac{2 e^{-2 H}}{1+e^{-2 H}}\right| \\
& \leqq 2 \sum_{k=1}^{\infty} e^{-2 k H}\left(e^{2 k\|V\|_{\bar{\alpha}}}-1\right) \\
& \leqq 2 \sum_{k=1}^{\infty} e^{-2 k H}\left(e^{2 k\|V\|_{1+2 \bar{\alpha}}}-1\right) .
\end{aligned}
$$

(A.14) Theorem. Under the assumptions of Theorem (3.10) each real $z_{0} \in U$ is contained in an open set $U_{z_{0}} \subset U$ on which (3.3) and (3.4) hold.

Proof. Fix a real $z_{0} \in U$, set $H=J_{\{0\}}\left(z_{0}\right)$ and

$$
J_{R}=\left\{\begin{array}{lll}
J_{R}\left(z_{0}\right) & \text { if } & |R| \geqq 2 \\
0 & \text { if } & |R|=1
\end{array}\right.
$$

We do the proof under the assumption that $H>0$.

One easily checks from (3.12) that

(A.15) $H>\sum_{R \ni 0}\left|J_{R}\right| 3^{|R|-1}$,

and thus the hypotheses of Lemma (A.7) are satisfied.

We also have from (3.12) that

(A.16) $\sum_{k=1}^{\infty} e^{-2 k H}\left(\exp \left[2 k \sum_{R \ni 0}\left|J_{R}\right| 3^{|R|-1}\right]-1\right)=b<1$.

Now the continuity of each $J_{R}(z)$ together with (3.11) implies that

$$
\sum_{\substack{R \ni 0 \\|R| \geqq 2}}\left|J_{R}(z)\right| 3^{|R|-1}+\left|J_{\{0\}}(z)-J_{\{0\}}\left(z_{0}\right)\right|
$$


is continuous on $U$; and thus, from (A.15), we see that there is an open $\tilde{U} \subset U$ containing $z_{0}$ such that

$$
v=\sup _{z \in \widetilde{U}} \sum_{\substack{R \neq 0 \\|R| \geqq 2}}\left|J_{R}(z)\right| 3^{|R|-1}+\left|J_{\{0\}}(z)-J_{\{0\}}\left(z_{0}\right)\right|<H .
$$

Now let

$$
U_{z_{0}}=\left\{z \in \tilde{U}: \sum_{R \ni 0}\left|J_{R}(z)-J_{R}\left(z_{0}\right)\right| 3^{|R|-1}<\frac{(1-b)(1+\exp [2(v-H)])^{2}}{8 \exp [2(v-H)]}\right\} .
$$

Again because of the continuity of each $J_{R}(z)$ and (3.11), $U_{z_{0}}$ is open and clearly contains $z_{0}$.

We now let $\alpha$ be as in the conclusion of Lemma (A.7) and $a=(1+b) / 2$ and check that (3.3) and (3.4) hold on $U_{z_{0}}$. Because of (0.1) it suffices to check them for $k=0$. Let

$$
V(z, \eta)=\sum_{R \ni 0} J_{R}(z) \prod_{j \in R \backslash\{0\}} \eta_{j}-H .
$$

Then $C_{0}(z, \eta)=1+\alpha \eta_{0}+\eta_{0}(\tanh (V(z, \eta)+H)-\alpha)$. But (A.17) implies that for all $z \in U_{z_{0}}, H>|V(z, \eta)|$. Therefore by Lemma (A.5), just as in (A.11), we have

(A.18) $\tanh (V(z, \eta)+H)-\alpha$

$$
\begin{aligned}
& =1-\alpha-\frac{2 e^{-2 H}}{1+e^{2-H}}+2 \sum_{k=1}^{\infty}(-1)^{k} e^{-2 k H}(\exp [-2 k V(z, \eta)]-1) \\
& =\tanh \left(V\left(z_{0}, \eta\right)+H\right)-\alpha \\
& +2 \sum_{k=1}^{\infty}(-1)^{k} e^{-2 k H}\left(\exp [-2 k V(z, \eta)]-\exp \left[-2 k V\left(z_{0}, \eta\right)\right]\right) .
\end{aligned}
$$

According to Lemma (A.7) the $\alpha$ norm of the first term on the right side of (A.18) is bounded by

$$
2 \sum_{k=1}^{\infty} e^{-2 k H}\left(\exp \left[2 k\left\|V\left(z_{0}, \cdot\right)\right\|_{1+2 \alpha}\right]-1\right) .
$$

By using Lemmas (A.1) and (A.4) and the increase of $\|V(z, \cdot)\|_{\gamma}$ as a function of $\gamma \geqq 1$, it is easily checked that the $\alpha$ norm of the second term is bounded by

$$
4 \sum_{k=1}^{\infty} k \exp [2 k(v-H)]\left\|V(z, \cdot)-V\left(z_{0}, \cdot\right)\right\|_{3},
$$

which is less than $(1-b) / 2$ for $z \in U_{z_{0}}$. Applying these two bounds to (A.18) we obtain

$$
\text { (A.19) } \begin{aligned}
& \frac{1}{1+\alpha}\|\tanh (V(z, \cdot)+H)-\alpha\|_{\alpha} \\
& \leqq \frac{1}{1+\alpha}\left(\frac{1-b}{2}+2 \sum_{k=1}^{\infty} e^{-2 k H}\left(\exp \left[2 k\left\|V\left(z_{0}, \cdot\right)\right\|_{1+2 \alpha}\right]-1\right)\right) \\
& \leqq \frac{1-b}{2}+2 \sum_{k=1}^{\infty} e^{-2 k H}\left(\exp \left[2 k\left\|V\left(z_{0}, \cdot\right)\right\|_{1+2 \alpha}\right]-1\right) /(1+\alpha)
\end{aligned}
$$


Since $V\left(z_{0}, \cdot\right)$ does not have a constant term when expanded in terms of the $\chi_{F}^{0}$ s, it follows that $\left(\exp \left[2 k\left\|V\left(z_{0}, \cdot\right)\right\|_{1+2 \alpha}\right]-1\right) /(1+\alpha)$ is an increasing function of $\alpha$. Thus, using (A.16), we may bound the right side of (A.19) by

$$
\frac{1-b}{2}+\sum_{k=1}^{\infty} \exp [-2 k H]\left(\exp \left[2 k\left\|V\left(z_{0}, \cdot\right)\right\|_{3}\right]-1\right)=a,
$$

proving (3.3).

To prove (3.4) note that (A.17) implies that for $z \in U_{z_{0}} \subset \tilde{U}$

$$
\begin{aligned}
H> & \sum_{\substack{R \ni 0 \\
|R| \geqq 2}}\left|J_{R}(z)\right| 3^{|R|-1}+\left|J_{\{0\}}(z)-H\right| \\
& \geqq\left|\sum_{R \ni 0} J_{R}(z) \prod_{j \in R \mid\{0\}} \eta_{j}-H\right| .
\end{aligned}
$$

The analyticity of $\gamma(z, 0, G)$ follows from (3.11), (3.7), (4.20), and the dominated convergence theorem. The uniform convergence of $\sum_{G}|\gamma(z, 0, G)|, z \in U_{z_{0}}$ is proved as in Theorem (A.2) using the first equality in (A.18).

\section{References}

1. Dobrushin, R.L.: Markov processes with a large number of locally interacting componentsexistence of the limiting process and its ergodicity. Probl. Peredaci Inform. 7, 70-87 (1971)

2. Dobrushin, R.L.: The description of a random field by means of conditional probabilities and conditions of its regularity. Theory Prob. Appl. 13, 197-224 (1968)

3. Holley, R., Stroock, D.: A martingale approach to infinite systems of interacting processes, to appear in Ann. Prob.

4. Holley, R., Stroock,D.: $L_{2}$ theory for the stochastic Ising model. Z. Wahrscheinlichkeitstheorie verw. Gebiete 35, 87-101 (1976)

5. Liggett, T.M.: Existence theorems for infinite particle systems. Trans. Amer. Math. Soc. 165, $471-481(1972)$

6. Sullivan, W.G.: A unified existence and ergodic theorem for Markov evolution of random fields. Z. Wahrscheinlichkeitstheorie verw. Geb. 31, 47-56 (1974)

7. Israel,R. B.: High-Temperature Analyticity in Classical Lattice Systems. Commun. math. Phys., in press (1976)

Communicated by J. L. Lebowitz

Received October 31, 1975

Note Added in Proof. The authors have recently received a preprint of [7] which contains another proof of Theorem (3.8) together with analogous results for other models. 
